\title{
Amphotericin B Assembles into Seven-Molecule Ion Channels in
}

\section{Membrane Domain}

\author{
Yuichi Umegawa ${ }^{1,2,10}$, Tomoya Yamamoto ${ }^{1,8,10}$, Mayank Dixit ${ }^{3,10}$, Kosuke Funahashi ${ }^{3}$, Sangjae \\ $\mathrm{Seo}^{3}$, Yasuo Nakagawa ${ }^{1}$, Taiga Suzuki ${ }^{1}$, Shigeru Matsuoka ${ }^{1,4,9}$, Hiroshi Tsuchikawa ${ }^{1,9}$, Shinya \\ Hanashima $^{1}$, Tohru Oishi ${ }^{1,5}$, Nobuaki Matsumori ${ }^{1,5}$, Wataru Shinoda ${ }^{3,6,7, *}$, Michio Murata ${ }^{1,2,4, *}$
}

\section{Affiliations:}

${ }^{1}$ Department of Chemistry, Graduate School of Science, Osaka University, Toyonaka, Osaka 560-0043,

10 Japan.

${ }^{2}$ Project Research Center for Fundamental Sciences, Osaka University, Toyonaka, Osaka 560-0043, Japan.

${ }^{3}$ Department of Materials Chemistry, Graduate School of Engineering, Nagoya University, Nagoya 4648603, Japan.

${ }^{4}$ Japan Science and Technology Agency, ERATO, Lipid Active Structure Project, Osaka University, Toyonaka, Osaka 560-0043, Japan.

${ }^{5}$ Department of Chemistry, Graduate School of Science, Kyushu University, Fukuoka 819-0395, Japan. ${ }^{6}$ Research Institute for Interdisciplinary Science, Okayama University, Okayama 700-8530, Japan. ${ }^{7}$ Department of Chemistry, Faculty of Science, Okayama University, Okayama 700-8530, Japan. 
*Corresponding author. Email: murata@chem.sci.osaka-u.ac.jp (M. M.) and shinoda@okayama-u.ac.jp (W. S.)

${ }^{10}$ These authors contributed equally to this work. ${ }^{8}$ Present address: Riken, Saitama 351-0198, Japan. ${ }^{9}$ Present address: Oita University, Oita 879-5593, Japan.

Abstract: Amphotericin $\mathrm{B}^{1,2}$, a long-used antifungal drug ${ }^{3}$, forms fungicidal ion-permeable channels across cell membranes. Using solid-state nuclear magnetic resonance spectroscopy and molecular dynamics simulations, we experimentally elucidated the three-dimensional structure of the molecular assemblies formed by this drug in membranes in the presence of the fungal sterol, ergosterol. A stable assembly of seven drug molecules was observed to form an ion conductive channel. The structure somewhat resembled the upper half of the barrel-stave model proposed in the $1970 \mathrm{~s}^{4}$ but different substantially in the number of molecules and their arrangement. Based on the structure obtained, the aggregation of the channel assemblies in membranes was investigated and a mechanism was proposed in which complexation with ergosterol stabilizes the drug's assemblies, leading to their aggregation, and in turn enhancing channel activity. The highresolution structure is consistent with many previous findings, including structure-activity relationships of the drug, and the channel aggregation provides a more reasonable explanation for the selective toxicity of this drug to fungi. 
The antifungal antibiotic amphotericin $\mathrm{B}(\mathrm{AmB}$, Fig. $1 \mathrm{~A})$ is produced by the bacterium Streptomyces nodosus, which was isolated from the soil of the Orinoco River in Venezuela ${ }^{1,2}$. $\mathrm{AmB}$ has been used for over 60 years to treat systemic fungal infections and remains one of the most clinically important antibiotics ${ }^{3,5,6}$. AmB penetrates the blood-brain barrier and thus serves as a standard therapeutic for most fungal infections of the central nervous system ${ }^{7}$. However, serious adverse effects of $\mathrm{AmB}$, such as nephrotoxicity, often result in discontinuation of therapy for life-threatening systemic fungal infections.

The self-assembly of bioactive molecules, such as antimicrobial peptides, in cell membranes has long been a subject of structural biology research ${ }^{8}$. However, despite their pharmacological importance, little is known about the self-assembled structures of nonpeptide natural products such as AmB, mainly due to the lack of a suitable method for structure determination and the difficulties in isotope labelling of the aperiodic and diverse compounds necessary for NMR measurements ${ }^{9}$. AmB self-assembles into an oligomeric structure in the ergosterol (Erg)containing membranes of fungal and yeast cells; this oligomer acts as an ion channel and is thought to be responsible for the selective toxicity of the drug against eukaryotic microbes ${ }^{5,9-11}$. However, 50 years after the well-known barrel-stave model $^{4}$ was proposed (Fig. 1C), the structure of the AmB channel has not been determined. The AmB-AmB interactions and functional groups involved in channel formation provide essential information for structural modifications to improve efficacy and reduce the toxicity of this important antibiotic. Thus, we aimed to determine the entire structure of the AmB channel assembly in Erg-containing membranes and its functionality as follows: 1) determine the number of AmB molecules per assembly by solid-state nuclear magnetic resonance (NMR) spectroscopy; 2) determine the interatomic distances between ${ }^{13} \mathrm{C}$-labelled $\mathrm{AmB}$ and ${ }^{19} \mathrm{~F}$-labelled $\mathrm{AmB}$ in the assembly by ${ }^{13} \mathrm{C}$ ${ }^{19} \mathrm{~F}$ rotational echo double resonance (REDOR) experiments; 3 ) determine the arrangement of 
AmB molecules in the assembly by using ${ }^{13} \mathrm{C}-{ }^{19} \mathrm{~F}$ distances to obtain a time-averaged structure of the assembly; 4) deduce the structural dynamics of the assembly by means of molecular dynamics (MD) simulations based on the time-averaged structure; and 5) estimate the ion permeability of the fluctuating assemblies by MD simulations and compare it with previous experimental results.

A
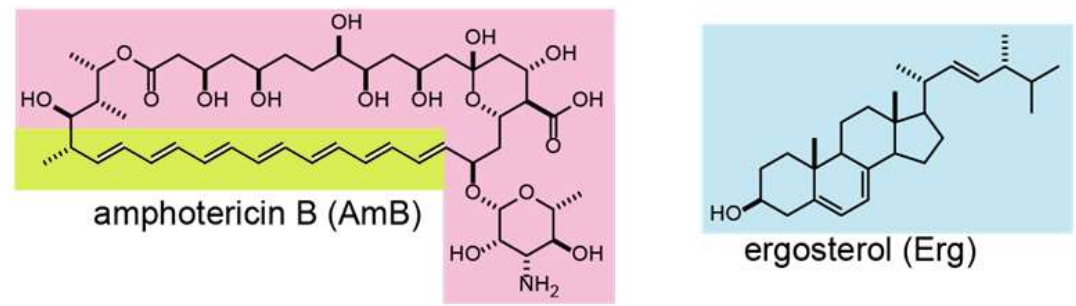

B
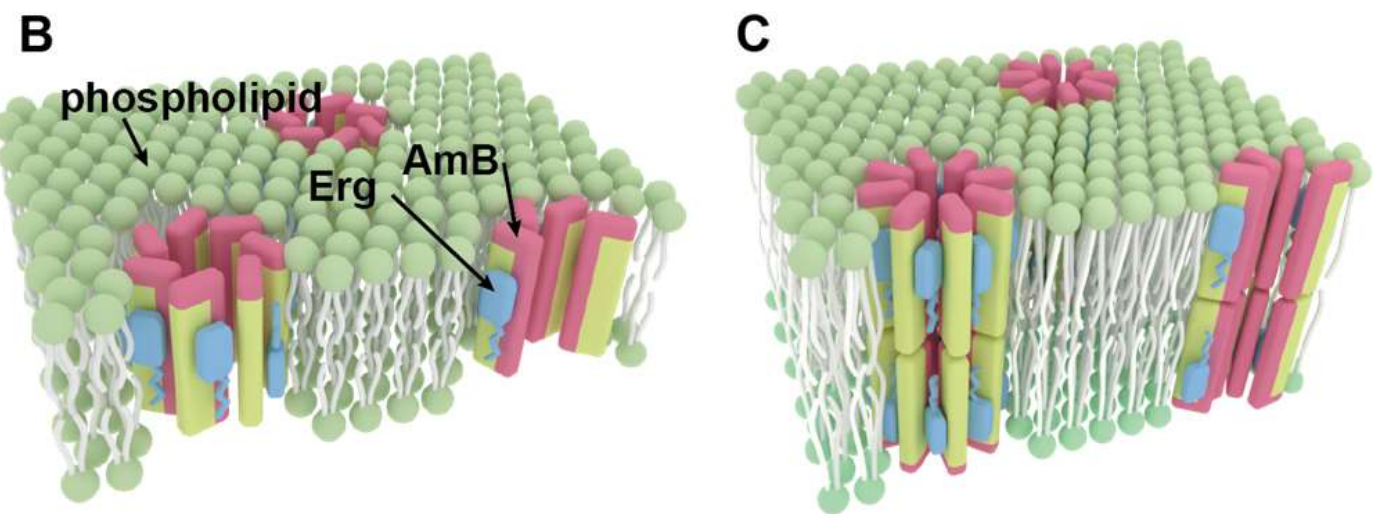

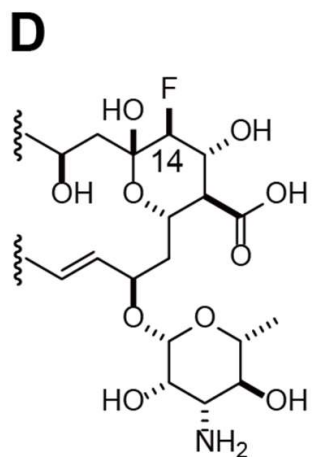

$14-\mathrm{F}-\mathrm{AmB}$
E

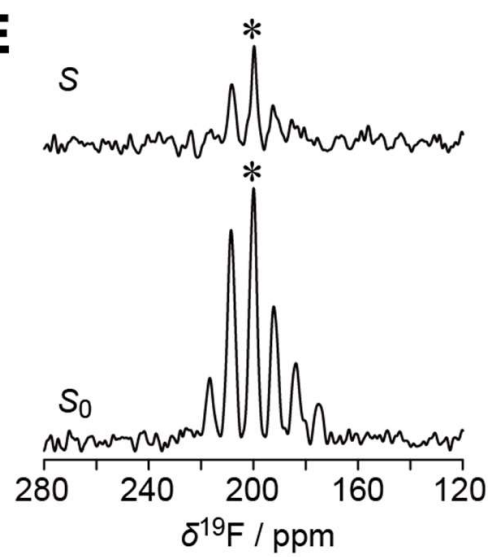

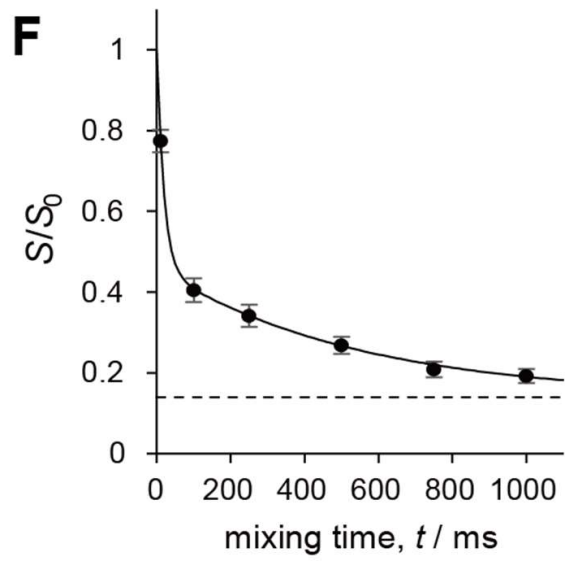


Chemical structures of AmB and Erg. (B) The present model for channel assemblies. (C) The classic barrel-stave model of the AmB ion channel proposed by De Kruijff et al. ${ }^{4}$ The hydrophobic and hydrophilic regions of $\mathrm{AmB}$ are depicted in greenish yellow and pale magenta, respectively. (D) Partial structure of 14-F-AmB. (E) $S$ (top) and $S_{0}$ (bottom) spectra of a 1:3:7 mixture of $14-F-A m B-E r g-P O P C$ in a ${ }^{19} \mathrm{~F}-\mathrm{CODEX}$ experiment (mixing time: $750 \mathrm{~ms}$ ). The spectra of the sample were obtained at $0^{\circ} \mathrm{C}$ by applying the magic angle spinning method with a rotation speed of $7 \mathrm{kHz}$. The signal in the central band marked by an asterisk (*) corresponds to the signal of the AmB remaining outside the membrane (see Fig. S5). (F) The decay curve of $S / S_{0}$, which denotes the fraction of the initial magnetization retained after mixing time. Error bars denote the noise level of the spectra obtained from integration of the baseline near the ${ }^{19} \mathrm{~F}$ signals. The intensity decayed according to the following biexponential equation: $S / S_{0}=0.14+$

$0.54 \mathrm{e}^{(-t / 19.1)}+0.32 \mathrm{e}^{(-t / 541.1)}$. This curve asymptotically approaches 0.14 (dotted line), indicating that 14-F-AmB forms a cluster of seven molecules.

\section{The number of AmB molecules constituting one channel assembly}

The average number of $\mathrm{AmB}$ molecules per channel assembly is crucial information for determining the 3D structure of AmB channels. Previously, this number has been estimated via channel conductance measurements ${ }^{12,13}$ and MD calculations ${ }^{14}$, but the results have varied widely from four to more than ten. Fluorine-labelled AmB (Fig. 1D), which is easily detected in NMR measurements, shows almost the same biological activity as unlabelled AmB (Fig. S1 and Table S1), and a method for its efficient preparation from natural products has been established ${ }^{15}$. Using the ${ }^{19} \mathrm{~F}$ centre band-only detection of the exchange (CODEX) method, which is often used to determine the oligomer number of molecules in assemblies ${ }^{16,17}$, we attempted to 
directly determine the number of AmB molecules per channel assembly (Fig. 1E). After removing the influence of unbound AmB (Fig. S4), we measured the $S / S_{0}$ values. The experimental values were plotted as a function of mixing time and found to lie on a curve that asymptotically approached 0.14 , which is the reciprocal of seven (Fig. 1F). These results implied that the average number of $\mathrm{AmB}$ molecules per assembly is seven because the magnetization of one $\mathrm{AmB}$ molecule diffuses equally towards the other neighbouring molecules in the assembly during the long mixing time. However, the results do not rule of the out the possibility that assemblies with six or eight molecules coexist within the membrane.

\section{Structural overview of AmB assembly in membranes}

We next determined the interatomic distances between adjacent AmB molecules in the assembly using solid-state NMR (Fig. 2). Skipped ${ }^{13} \mathrm{C}$-labelled AmB was obtained by a biosynthetic method (Figs. 2C, M7). AmBs fluorinated at positions 14 or 32 and $26,40-{ }^{13} \mathrm{C}_{2}-$ AmB (Fig. 2C) were prepared by chemical synthesis ${ }^{15,18}$ (Supplementary Materials and Methods). Two lipid bilayer models with different lipid compositions and different Erg concentrations in palmitoyloleoylphosphatidylcholine (POPC) were used for ${ }^{13} \mathrm{C}-{ }^{19} \mathrm{~F}$ rotational echo double resonance (REDOR, Fig. 2A) experiments because a low concentration of Erg was needed to measure REDOR for $26,40-{ }^{13} \mathrm{C}_{2}-\mathrm{AmB}$ due to signal overlap with Erg (Figs. 2A and S2). Six parameters (Figs. 3A, 3B and Figs. S3 and S4) were determined to elucidate the 3D structure of the AmB channel assembly: the number of molecules $n$ per assembly, the Euler angles $(\alpha, \beta, \gamma)$, the channel inner diameter $R$, and the adjacent probability $p$, as described in Fig. S4. Therefore, the distance was measured by using as many labelled atom pairs as possible; we adopted five REDOR-derived distances (Fig. 2B) plus the CODEX result to determine the 
parameters. For the REDOR experiment, time-dependent increases in the $\Delta S / S_{0}$ values were observed for each ${ }^{13} \mathrm{C}-{ }^{19} \mathrm{~F}$ pair (Fig. 2B), indicating that molecular motion was sufficiently slow to allow accurate measurements of the ${ }^{13} \mathrm{C}-{ }^{19} \mathrm{~F}$ distances in the membrane. With these distance constraints in hand, we investigated an assembly structure that could account for the NMR results without being constrained by previous models, such as the barrel-stave model and sterolsponge mode ${ }^{19}$. Recently, we found that the vertical penetration of AmB through the lipid bilayer corresponds to the length of a single drug molecule under experimental conditions similar to those used in this study ${ }^{20,21}$. The present REDOR experiment showed that the AmB molecules in the assembly are arranged in a parallel manner, as assumed in the classic barrel-stave model (Fig. S6). Thus, the results strongly suggest that the AmB assembly resembles the upper half of the barrel-stave model (Figs. 1B and S7), and other possible structures, such as the sterol-sponge model, are considered extremely unlikely. 

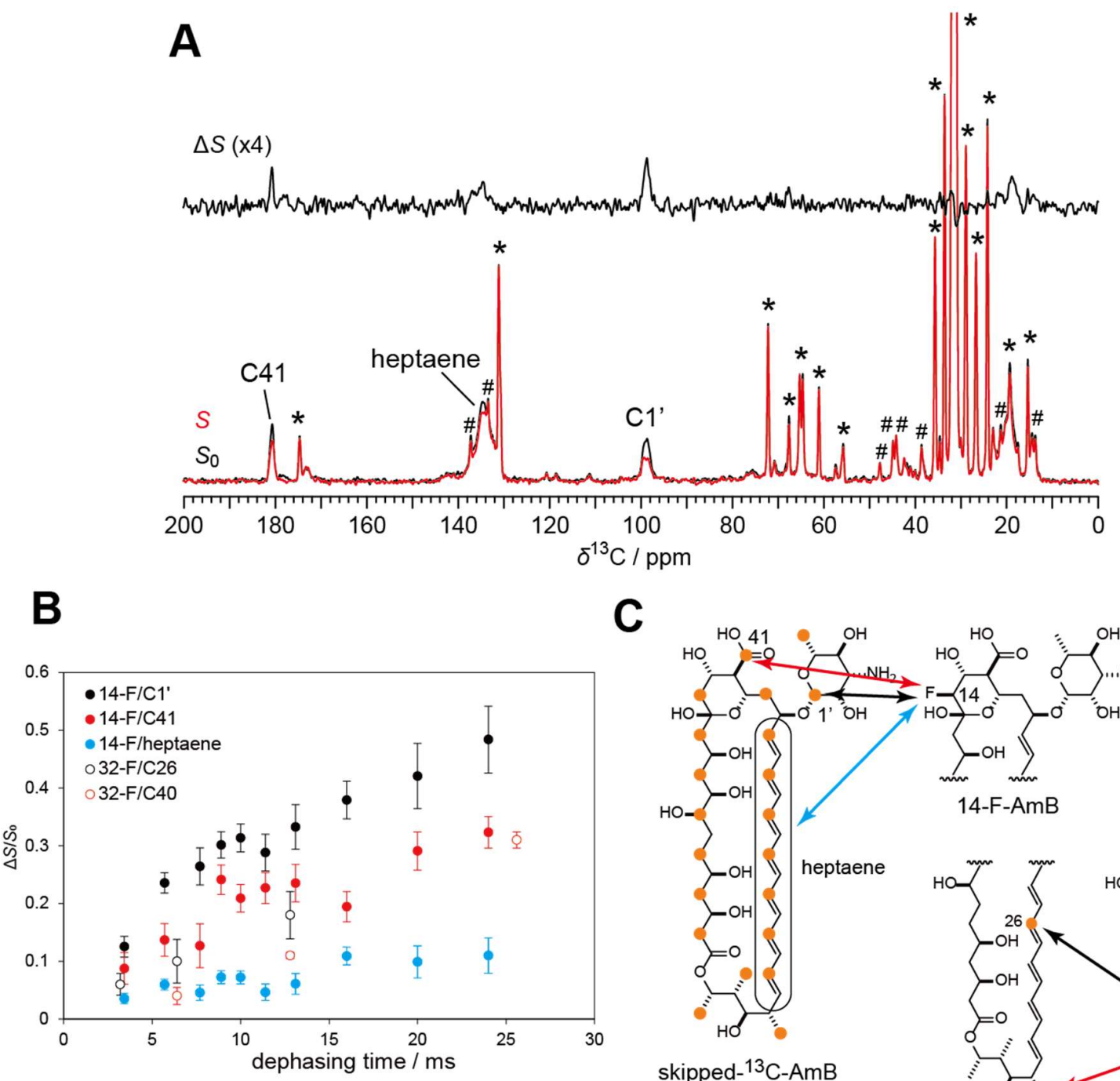

C

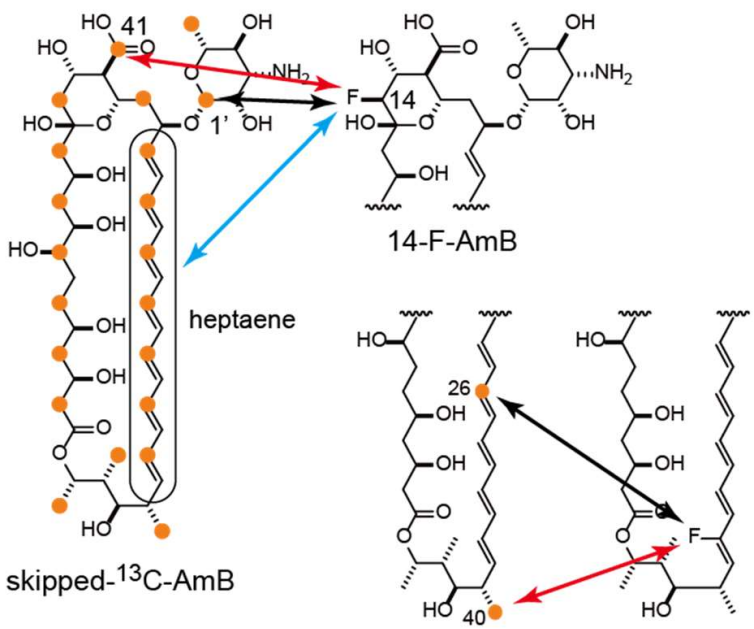

$26,40-{ }^{13} \mathrm{C}_{2}-\mathrm{AmB}$

32-F-AmB

Fig. 2. Solid-state NMR experiments used to determine intermolecular ${ }^{13} \mathrm{C}-{ }^{19} \mathrm{~F}$ distances.

(A) ${ }^{13} \mathrm{C}\left\{{ }^{19} \mathrm{~F}\right\}$ REDOR spectra of $14-\mathrm{F}-\mathrm{AmB} /$ skipped- ${ }^{13} \mathrm{C}-\mathrm{AmB} / \mathrm{Erg} / \mathrm{POPC}=0.5 / 0.5 / 3 / 7$ hydrated

5 with $10 \mathrm{mM}$ HEPES containing $\mathrm{D}_{2} \mathrm{O}$ at $25^{\circ} \mathrm{C}$. The sample spinning speed was $7 \mathrm{kHz}$, and the dephasing time was $16 \mathrm{~ms}$. The full echo ( $S_{0}$, bottom, black), REDOR-dephased ( $S$, bottom, red), and difference $(\Delta S$, top) spectra clearly showed a reduction in the peak intensity of the $\mathrm{C} 41$, heptane and $\mathrm{Cl}$ ' signals; * and \# indicate the signals attributed to POPC and Erg, respectively. (B) REDOR dephasing observed for C1' (filled black), C41 (filled red), and heptaene (filled blue) upon irradiation at 14-F and for C26 (open black) and C40 (open red) upon irradiation at 
32-F. The error bars denote the $\mathrm{S} / \mathrm{N}$ ratios of $\Delta S$ signals. (C) ${ }^{13} \mathrm{C}$-labelled positions of the skipped labelled and synthetic AmBs shown with orange circles. Arrows denote the interatomic distances determined by REDOR and are coloured in the same manner as the dots in (B).

\section{Time averaged structure of the AmB assembly revealed by NMR}

We generated a time-averaged structure of the AmB assembly as follows: the crystal structure ${ }^{22}$ was adopted for the conformation of an $\mathrm{AmB}$ molecule and the radial arrangement of the molecules was used as a starting point to search for the best structure that reproduced the distance constraints (Table S2) ${ }^{23}$. We varied the six parameters that defined the structure to minimize the root mean square deviation (RMSD) between the interatomic distances in the assembly model and the REDOR-derived distances (Figs. S4, S8). Fig. 3C shows curves of the experimental $\Delta S / S_{0}$ values obtained for each interatomic distance in the AmB assembly as a function of the dephasing time. For the interatomic $14-\mathrm{F} / \mathrm{C} 41,14-\mathrm{F} / \mathrm{C} 1$ ' and $32-\mathrm{F} / \mathrm{C} 40$ distances, two distances, $r_{1}$ and $r_{2}$ (Fig. 3B), were obtained for each labelled carbon atom; the 32-F/C26 distance deviated slightly from the measured value, presumably because of an interaction between assemblies as described below. This is the first experimentally validated structure of $\mathrm{AmB}$ assembly in a membrane (Fig. 3D). In the structure, the molecular axis of AmB is nearly perpendicular to the membrane plane (the cone angle $\beta$ is approximately $2^{\circ}$ ), and the channel radius at the oxygen atom on $\mathrm{C} 8$ is $5.4 \AA$. 

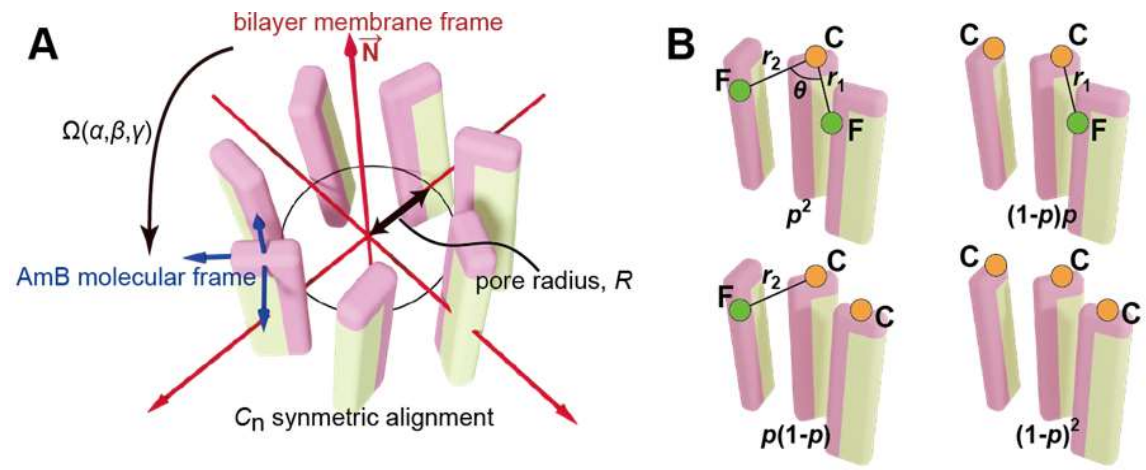

C
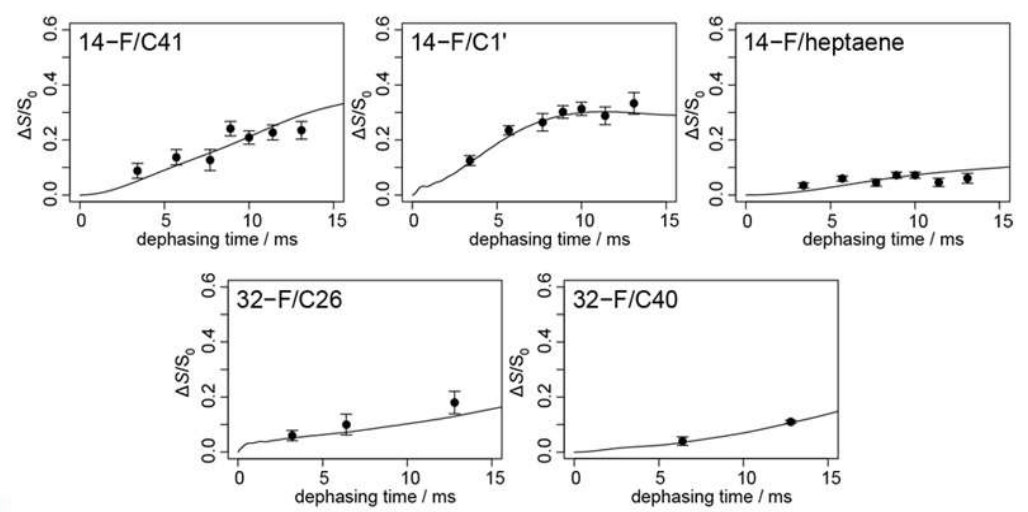

D

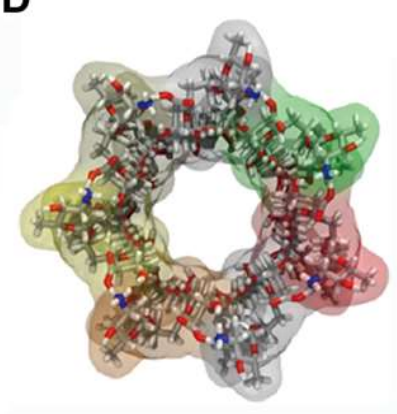

E
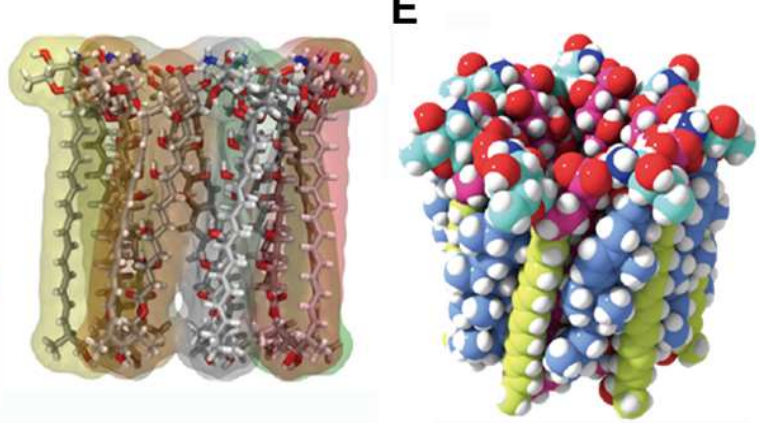

Fig. 3. Construction of a symmetric structure of the AmB assembly based on the

interatomic distances obtained via NMR. (A) Definition of the parameters $\alpha, \beta, \gamma, n$, and $R$ (see Fig. S4). (B) Possible adjacent combinations of ${ }^{19} \mathrm{~F}-\mathrm{AmB}$ and ${ }^{13} \mathrm{C}-\mathrm{AmB}$ and the abundance ratios for the probability parameter $p$ (Fig. S3). (C) Simulated REDOR dephasing curves calculated from the best fitted parameters $(\alpha, \beta, \gamma, n, R$, and $p)=\left(-12^{\circ}, 2^{\circ},-20^{\circ}, 7,5.4 \AA\right.$, and 0.24$)$ and experimental dephasing values (same as Fig. 2B). (D) Top and side views of the symmetric structure of the heptamer (seven-molecule) assembly. (E) Space-filling model of the seven AmB and seven Erg complexes. 


\section{Dynamic structure of the AmB assembly obtained from MD simulations}

To clarify the structure of a functional ion channel from the AmB-AmB configuration derived from NMR, we next simulated the molecular dynamics of the AmB assembly using MD

calculations. The timescale of the REDOR experiment is in milliseconds, which is much slower than the molecular fluctuations occurring in the submicrosecond range in lipid bilayers.

Therefore, the magnitude of REDOR dephasing reflects the time-averaged ${ }^{13} \mathrm{C}-{ }^{19} \mathrm{~F}$ distances in the AmB assembly, and these distances correspond to a static structure with centrosymmetry (Fig. 3D, E). Knowing how the channel structure varies with time is vital to more fully understanding the molecular mechanisms of ion flux, which is responsible for the pharmacological activities of AmB. An accurate initial structure (Fig. 4A) is essential for simulating a dynamic structure because the simulation time is limited to microseconds, but the exchange of interacting molecules in the assembly occurs much more slowly than that in the simulation. Thus, the following events cannot occur during the simulation time: a change in the number of AmB molecules per assembly, exchange between the single-length assembly (Fig. 1A) and the double length assembly (Fig. 1B), and flipping of the AmB or Erg structure. Figs. 4B and 4C show a typical snapshot and the average structure estimated from MD simulations, respectively. Each Erg molecule generally continues to interact with the same AmB molecule during the $1 \mu$ s simulation (Figs. 4B, 4D and S11). These results are consistent with a previous report that the AmB-Erg complex has a lifetime longer than the NMR timescale ${ }^{18}$. In addition, the averaged interatomic distances between isotope-labelled positions were calculated to directly compare the channel structures obtained from solid-state NMR to those obtained by MD simulations, and the results were in good agreement (Figs. 4E, S18-S20 and Table S3). 
The resulting structure shares some similarities with the classic model ${ }^{4}$ proposed in the 1970s, but significant differences were observed: the number of AmB molecules per channel assembly is seven rather than eight, the macrocycle plane has a spiral rather than radial configuration (Fig. 3D, 3E), and the length of the assembly that penetrates the lipid bilayer corresponds to the length of a single molecule rather than the length of two molecules (Figs. $1 \mathrm{~B} / \mathrm{C}$ and S12), even though the single-length assembly was assumed to be too short to function as an ion channel ${ }^{14}$. We also found that intermolecular hydrogen bonds from the amino sugar moieties in AmB-AmB and AmB-Erg provide an important force that stabilizes these ion channels in an active form (Fig. 4D, Table S5). 
A

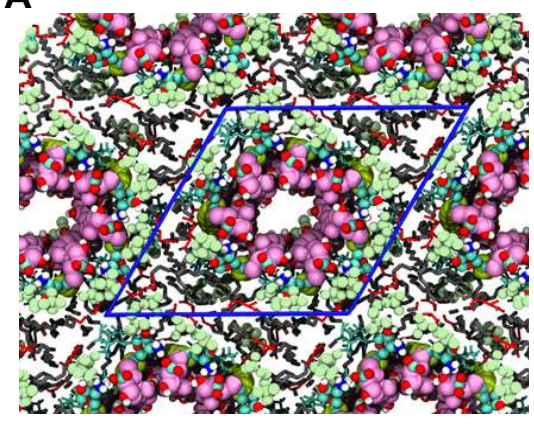

B

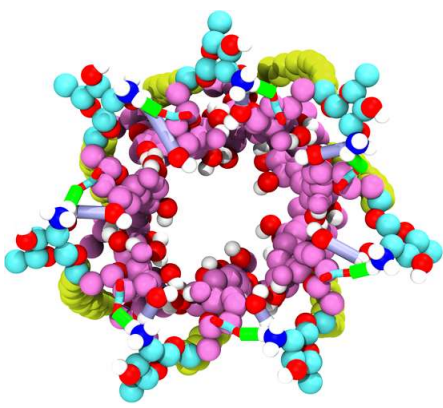

C
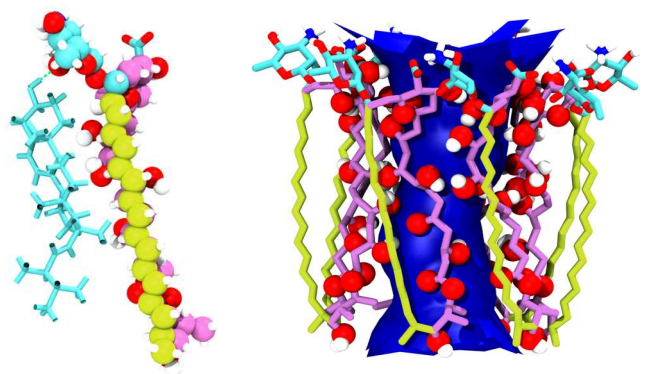

5

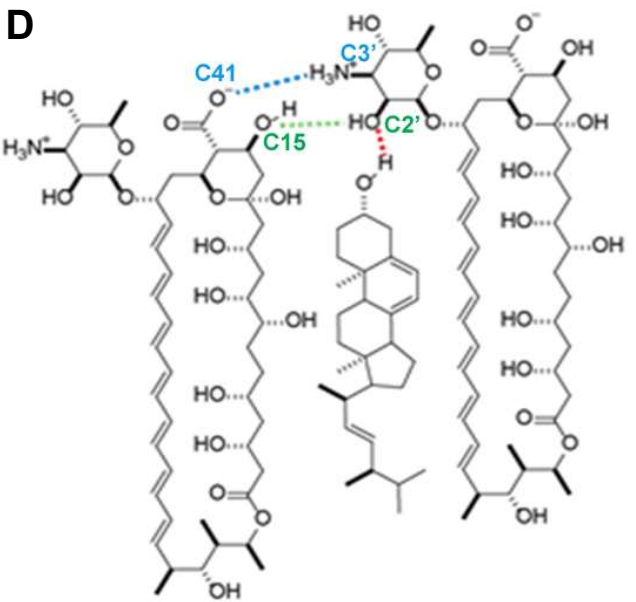

$\mathbf{E}$

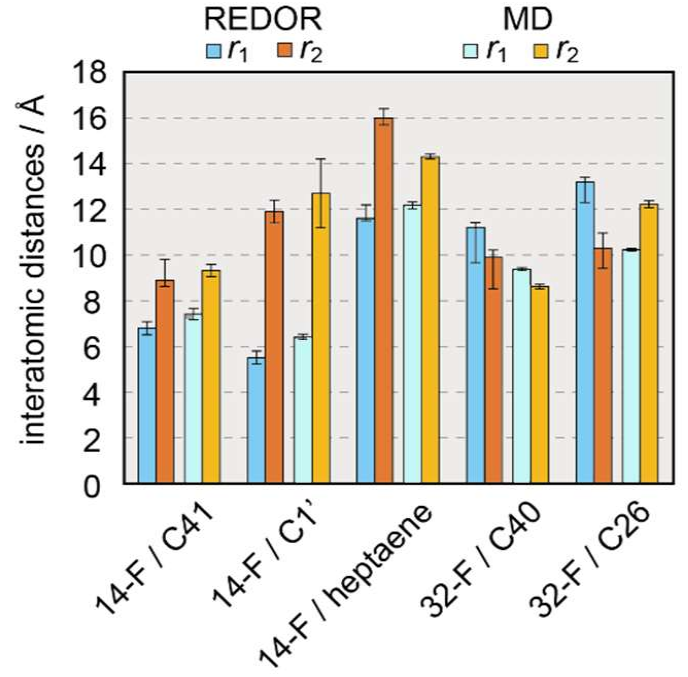

Fig. 4. Construction of an asymmetric assembly based on MD simulations. (A) Initial structure of the AmB assemblies with periodic boundary conditions constructed on the basis of NMR results. (B) Snapshots of all-atom MD simulations of an AmB assembly and an AmB-Erg complex. (C) Average structure of an AmB assembly showing the water-accessible region inside the pore. (D) Major intermolecular interactions stabilizing the AmB-Erg channel assembly. (E) Interatomic distances measured by solid-state NMR experiments and the values from MD simulations. The distances differ between the front and back of the molecule and correspond to $r 1$ and $r 2$ (Fig. 3B); the structure obtained by MD is not rotationally symmetric, so the average $\pm \mathrm{SD}$ is shown, where SD values obtained from ten runs of the simulation were less than $5 \%$. 


\section{Ion conduction activity of AmB and AME assemblies evaluated by MD simulations}

To evaluate the structural similarity between the AmB assembly constructed on the basis of NMR and MD results and the real ion channel responsible for drug activity, we measured the conductance of the assembly under conditions similar to those used for single channel-recording experiments ${ }^{24}$. The channel activity of the dynamic AmB assembly was estimated in Ergcontaining diphytanoylphosphatidylcholine (DPhPC) membranes with MD simulations (Figs. M6, S13, S22-S25 and Tables S6-S8). As shown in Fig. 5A and 5B, the heptamer channel best reproduced the experimental ion permeability ${ }^{24}$, and the formation of a water column in the channel pore was deduced to significantly stabilize the channel structure (Fig. 4C). Assemblies consisting of six or fewer $\mathrm{AmB}$ molecules did not allow $\mathrm{K}^{+}$passage, whereas assemblies of eight molecules allowed much more $\mathrm{K}^{+}$to pass through than was observed experimentally (Fig. 5A).

Accurately elucidating the channel structure enables us to explain some previously reported structure-activity relationships. For example, the destructive effect of $N$-acylation on the antifungal activity of $\mathrm{AmB}$ and the retained activity of AmB methyl ester (AME), which was used in human clinical trials, were demonstrated by MD simulations ${ }^{25}$ (Figs. S14, S28 and Tables S9, S10); the simulated channel activity of AME also reproduced previous experimental values $^{26}$ (Fig. 5A).

\section{Aggregation of AmB channel assemblies}

Next, we must consider the similarity between the present AmB channel formed in the model bilayers and the real ion channels that exert antifungal activity in the human body. The apparent difference in the channel formation conditions is the concentration of $\mathrm{AmB}$ in the membranes since the AmB concentration for NMR measurements was approximately 100 times that found in 
real fungal membranes ${ }^{6}$. First, we performed UV-VIS and CD spectroscopy to determine whether the 3D structure of the channel assembly changed with changes in the AmB concentration in the membrane. The UV-VIS spectra (Fig. S9) of the heptaene moiety of AmB in the Erg-containing membrane showed typical absorbance bands with sharper and redshifted peaks, distinct from those in the spectra of Cho-containing or sterol-free membranes. These features were unaffected by the concentration of $\mathrm{AmB}$, indicating that the heptaene moiety resided stably in the membrane interior at concentrations ranging from $0.1 \mathrm{~mol} \%$ to $10 \mathrm{~mol} \%$ of the total lipid concentrations ${ }^{27}$. The $\mathrm{CD}$ spectra of $\mathrm{AmB}$, which sensitively reflect the molecular arrangements of $\mathrm{AmB}$ in membranes ${ }^{28}$, showed that $\mathrm{AmB}$ formed essentially the same assembly over the same concentration range used for the UV-VIS measurements (Fig. S10). Therefore, we presume that the structure of AmB assemblies elucidated by solid-state NMR and MD is basically the same as that of ion channels occurring in fungal membranes at low AmB concentrations. Usually, peptides maintain a monomeric form at very low membrane concentrations and form assemblies with a larger number of molecules at higher concentrations $^{29}$. Why do AmB molecules form a similar assembly despite the large concentration difference? We speculate that this similarity can be explained by the stability of the heptamer assembly (Fig. S8) and the resulting aggregation of the channel assemblies. As shown in Figs. 5C and 5D (also Fig. S16), the number of unstable interdigitating lipids surrounding the assemblies is expected to be reduced by aggregation of the channels. Even if the overall $\mathrm{AmB}$ concentration is low, as in the case of antifungal assays, the AmB concentration is assumed to increase locally upon aggregation of the channel assemblies. This idea is also supported by the ${ }^{2} \mathrm{H}$ NMR spectra of deuterium-labelled AmB at various concentrations. We previously reported that assemblies of AmB methyl ester (AME) diffuse in lipid bilayers when highly diluted ${ }^{21}$. Conversely, AmB assemblies did not show significant diffusional movement 
even at an AmB-to-lipid ratio of $1 \mathrm{~mol} \%$ (Fig. S15), suggesting that AmB channels tend to form large aggregates in lipid bilayers. This aggregation can explain the stability of the single-length assembly in the model bilayers and possibly in fungal membranes.

In mammalian cell membranes containing cholesterol, the interaction between AmB and cholesterol is too weak to form aggregates of ion channels in Erg-membranes, often resulting in unstructured amorphous AmB molecules outside the membranes (Figs. S11 and S21), as revealed by the UV-VIS spectra ${ }^{20}$ (Figs. S9 and S17). Nevertheless, AmB channels occurring in mammalian cells could be structurally similar to those in Erg-containing membranes (Fig. S1 1 and Table S5) and thus weakly increase the ion permeability of the membrane, resulting in adverse drug effects. 
A

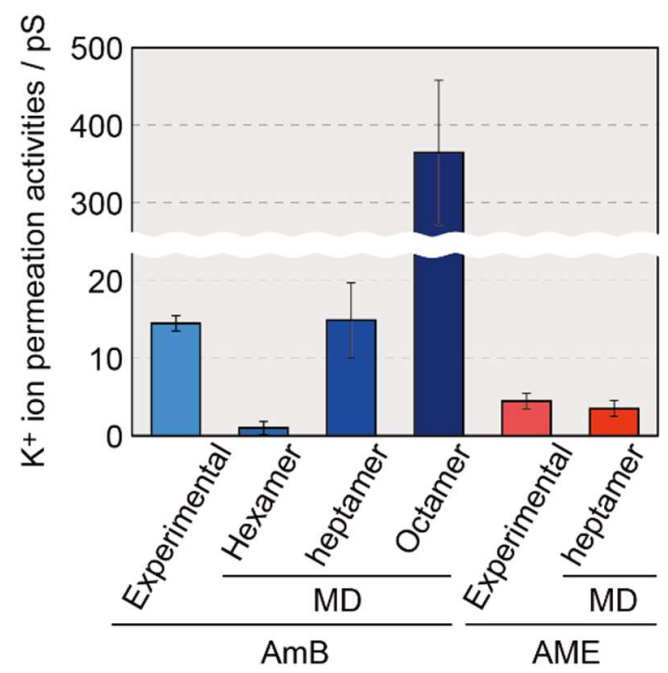

C

10

15

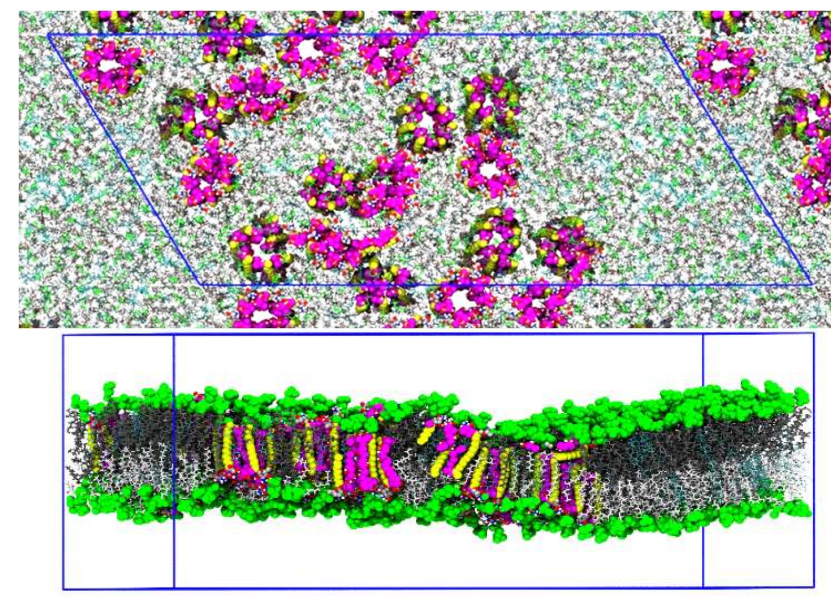

B

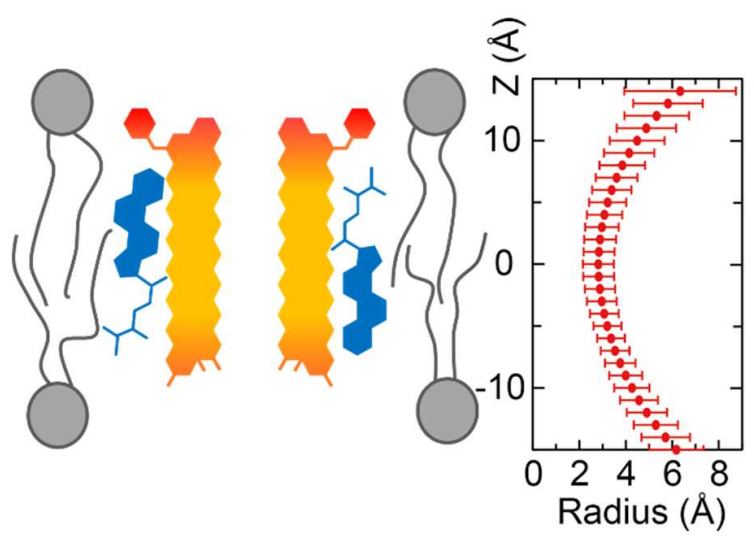

D

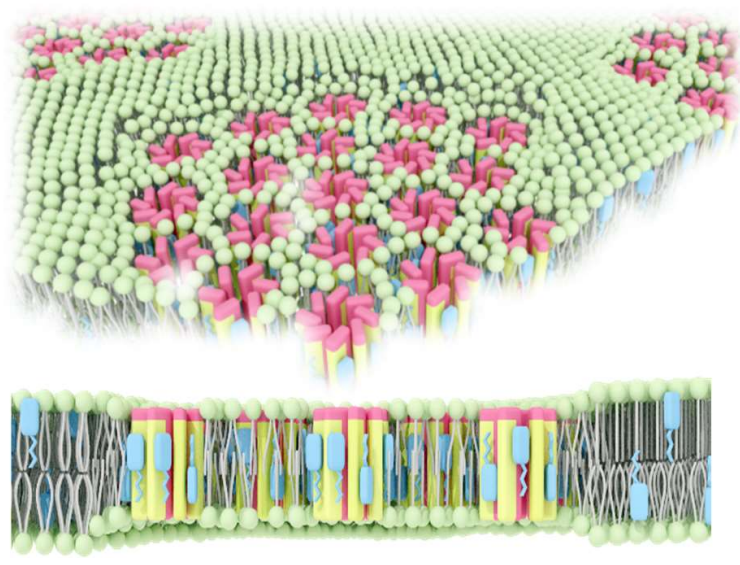

Fig. 5. Ion channel activity of an AmB assembly based on MD simulations and aggregate formation of AmB assemblies in the membrane. (A) Experimental and MD-derived values of $\mathrm{K}^{+}$permeability of $\mathrm{AmB}$ and $\mathrm{AME}^{24,26}$; experiments in the literature were carried out by using DPhPC-Erg 5:1 (2 M KCl, at $100 \mathrm{mV})^{24}$ and egg yolk lecithin ${ }^{26}$ membranes. This clearly indicates that the channel that shows a value close to those of past single channel measurements is the heptamer assembly. (B) Channel radius profile (distance from the centre of a water molecule) along the horizontal axis obtained for the present channel structure. The horizontal 
bars denote the standard deviation of the channel radius during the simulation. (C) Snapshot of the dimerization (partial aggregation) of AmB-Erg assemblies after $2 \mu$ s M simulation under conditions similar to those of NMR experiments containing the antiparallel channel-channel arrangement. The blue frame shows the periodic boundary box (see also Fig. S27). (D) Illustration of aggregates of channel assemblies formed in membrane, where tens of assemblies are aggregated with lipid interdigitations (see Fig. S26 for the MD simulation).

\section{Conclusion}

We elucidated the dynamic structure of the assembly using NMR experiments and MD simulations. To investigate whether this molecular assembly functions as an ion channel, the conductance of $\mathrm{K}^{+}$obtained by MD simulations was compared with the experimental values from single channel recordings. The two values agreed well, indicating that the structure of the AmB channel determined in this study is more accurate in terms of the number of molecules per channel and the mode of $\mathrm{AmB}-\mathrm{AmB}$ interaction than those previously proposed. The obtained structure allowed us to propose a mechanism in which the complexation of AmB with Erg stabilizes the assemblies and induces their aggregation, thereby enhancing ion channel activity. Erg induces this aggregation much more efficiently than cholesterol. This effect of Erg is possibly responsible for the selective toxicity of $\mathrm{AmB}$ to fungi. The present $3 \mathrm{D}$ structure of the AmB channel and its behaviour in sterol-containing membranes could, therefore, be useful for improving drug efficacy and reducing adverse effects.

\section{Online content}


Detailed MD simulations and synthetic methods, additional references, supplementary information, acknowledgements; details of author contributions and competing interests; and statements of data and code availability are available at https://doi.org/XXXXXX.

1. Donovick, R., Gold, W., Pagano, J. F. \& Stout, H. A. Amphotericins A and B, antifungal antibiotics produced by a streptomycete. I. In vitro studies. Antibio. Annu. 3, 579-586 (1956)

2. Stiller, E. T., Vandeputte, J. \& Wachtel, J. L. Amphotericins A and B, antifungal antibiotics produced by a streptomycete. II. The isolation and properties of the crystalline amphotericins. Antibiotics Annual. 3, 587-591 (1956).

3. Hartsel,S. \& Bolard, J. Amphotericin B: new life for an old drug. Trends Pharmacol. Sci. 17, 445-449 (1996).

4. de Kruijff, B. \& Demel, R. A. Polyene antibiotic-sterol interactions in membranes of Acholeplasma laidlawii cells and lecithin liposomes. 3. Molecular structure of the polyene antibiotic-cholesterol complexes. Biochim. Biophys. Acta 339, 57-70 (1974).

5. Bolard, J. How do the polyene macrolide antibiotics affect the cellular membrane properties? Biochim. Biophys. Acta 864, 257-304 (1986).

6. Ellis, D. Amphotericin B: spectrum and resistance. J. Antimicrob. Chemother. 49, 7-10 (2002).

7. Petraitis, V. et al. Amphotericin B penetrates into the central nervous system through focal disruption of the blood-brain barrier in experimental hematogenous Candida meningoencephalitis, Antimicrob. Agents Chemother. 63, e01626-19 (2019).

8. Luo, W. \& Hong, M. Determination of the oligomeric number and intermolecular distances of membrane protein assemblies by anisotropic ${ }^{1} \mathrm{H}$-driven spin diffusion NMR spectroscopy. J. Am. Chem. Soc. 128, 72427251 (2006)

9. Vertut-Croquin, A., Bolard, J. \& Gary-Bobo, C. M. Enhancement of amphotericin B selectivity by antibiotic incorporation into gel state vesicles. A circular dichroism and permeability study. Biochem. Biophys. Res. Commun. 125, 360-366 (1984).

10. Davis, S. A. et al. Nontoxic antimicrobials that evade drug resistance. Nat. Chem. Biol. 11, $481-487$ (2015). 
11. Dong, P. et al. Polarization-sensitive stimulated Raman scattering imaging resolves amphotericin B orientation in Candida membrane, Sci. Adv. 7, eabd5230 (2021).

12. Venegas, B., González-Damián, J., Celis, H. \& Ortega-Blake, I. Amphotericin B channels in the bacterial membrane: role of sterol and temperature. Biophys. J. 85, 2323-2332 (2003).

13. Gabrielska, J., Gagoś, M., Gubernator, J. \& Gruszecki, W. I. Binding of antibiotic amphotericin B to lipid membranes: A ${ }^{1} \mathrm{H}$ NMR study. FEBS Lett. 580, 2677-2685 (2006).

14. Zielińska, J. et al. Self-assembly, stability and conductance of amphotericin B channels: bridging the gap between structure and function. Nanoscale 13, 3686-3697 (2021).

15. Matsumori, N., Umegawa, Y., Oishi, T. \& Murata, M. Bioactive fluorinated derivative of amphotericin B. Bioorganic Med. Chem. Lett. 15, 3565-3567 (2005).

16. deAzevedo, E. R., Hu, W.-G., Bonagamba, T. J. \& Schmidt-Rohr, K. Centerband-only detection of exchange: Efficient analysis of dynamics in solids by NMR. J. Am. Chem. Soc. 121, 8411-8412 (1999).

17. Mani, R. et al. Membrane-dependent oligomeric structure and pore formation of a $\beta$-hairpin antimicrobial peptide in lipid bilayers from solid-state NMR. Proc. Natl. Acad. Sci. USA 103, 16242-16247 (2006).

18. Nakagawa, Y. et al. The structure of the bimolecular complex between amphotericin B and ergosterol in membranes is stabilized by face-to-face van der Waals interaction with their rigid cyclic cores. Biochemistry 55, 3392-3402 (2016).

19. Anderson, T. M. et al. Amphotericin forms an extramembranous and fungicidal sterol sponge. Nat. Chem.Biol. 10, 400-406 (2014).

20. Yamamoto, T. et al. The Amphotericin B-ergosterol complex spans a lipid bilayer as a single-length assembly. Biochemistry 58, 5188-5196 (2019).

21. Yamamoto, T. et al. The perpendicular orientation of amphotericin B methyl ester in hydrated lipid bilayers supports the barrel-stave model. Biochemistry 58, 2282-2291 (2019).

22. Jarzembska, K. N. et al. Controlled crystallization, structure, and molecular properties of iodoacetylamphotericin B. Cryst. Growth Des. 12, 2336-2345 (2012). 
23. Matsumori, N., Sawada, Y. \& Murata, M. Mycosamine orientation of amphotericin B controlling interaction with ergosterol: Sterol-dependent activity of conformation-restricted derivatives with an amino-carbonyl bridge. J. Am. Chem. Soc. 127, 10667-10675 (2005).

24. Brutyan, R. A. \& McPhie, P. On the one-sided action of amphotericin B on lipid bilayer membranes. J. Gen. Phisiol. 107, 69-78 (1996).

25. Chéron, M. et al. Quantitative structure-activity relationships in amphotericin B derivatives. Biochem. Pharmacoly. 37, 827-836 (1988).

26. Hartsel, S. C., Benz, S. K., Ayenew, W. \& Bolard, J. Na $\mathrm{K}^{+}$and $\mathrm{Cl}^{-}$selectivity of the permeability pathways induced through sterol-containing membrane vesicles by amphotericin B and other polyene antibiotics. Eur. Biophys. J. 23, 125-132 (1994).

27. Fujii, G., Chang, J. E., Coley, T. \& Steere, B. The formation of amphotericin B ion channels in lipid bilayers. Biochemistry 36, 4959-4968 (1997).

28. Vertut-Croquin, A., Bolard, J., Chabbert, M. \& Gary-Bobo, C. Differences in the interaction of the polyene antibiotic amphotericin B with cholesterol- or ergosterol-containing phospholipid vesicles. A circular dichroism and permeability study. Biochemistry 22, 2939-2944 (1983).

29. Huang, H. W. Molecular mechanism of antimicrobial peptides: The origin of cooperativity. Biochim. Biophys. Acta 1758, 1292-1302 (2006). 


\section{Materials and Methods}

\section{Materials}

AmB was purchased from Nacalai Tesque (Kyoto, Japan). Ergosterol (Erg) and cholesterol (Cho) were purchased from Tokyo Kasei (Tokyo, Japan). POPC was purchased from NOF Corp (Tokyo, Japan). [1${ }^{13} \mathrm{C}$ ] glucose was purchased from Cambridge Isotope Laboratory (Massachusetts, U. S. A.). Streptomyces nodosus was provided by the RIKEN BRC through the National Bio Resource Project of the MEXT/AMED, Japan. All other analytical-grade chemicals were purchased from standard sources. 14-F$\mathrm{AmB}$ was chemically derivatized from $\mathrm{AmB}^{14}$. Deuterated $\mathrm{AmB}$ at the terminal dimethyl moiety, 39,40$d_{6}$-AmB, was biosynthetically prepared as reported previously by using ${ }^{2} \mathrm{H}$-labelled acetic acid ${ }^{30}$. Skipped- ${ }^{13} \mathrm{C}-\mathrm{AmB}$ and $39,40-d_{6}$-AmB were biosynthetically prepared by feeding Streptomyces nodosus with $\left[1-{ }^{13} \mathrm{C}\right]$ glucose and sodium $\left[3-{ }^{2} \mathrm{H}_{3}\right]$ propionate, respectively ${ }^{30}$. Solution NMR spectra were measured by JEOL ECS400 or ECA500 (Tokyo, Japan). Mass spectra were recorded on Thermo Fisher Scientific Orbitrap XL (Massachusetts, U. S. A.).

Synthesis of $26,40-{ }^{13} \mathrm{C}_{2}-\mathrm{AmB}: 26,40-{ }^{13} \mathrm{C}_{2}-\mathrm{AmB}$ was prepared according to Scheme $\mathrm{S} 1$. The details of the synthesis are provided in Supplementary Materials.

Preparation of skipped-13C-AmB: Skipped-13C-AmB was prepared as follows. Streptomyces nodosus was cultured in the production media $(50 \mathrm{~mL})$ in a $500-\mathrm{mL}$ Erlenmeyer flask at $26^{\circ} \mathrm{C}$ with shaking at $200 \mathrm{rpm}$. The production media contains [1-13C] glucose $(5.0 \mathrm{~g} / \mathrm{L})$, dextrin $(15 \mathrm{~g} / \mathrm{L})$, soy bean flower $(7.5 \mathrm{~g} / \mathrm{L})$, and $\mathrm{CaCO} 3(10 \mathrm{~g} / \mathrm{L})$. Additional [1-13C] glucose (111 mg in $200 \mu \mathrm{L}$ of water) was pulse feed to the media after $7 \mathrm{~h}, 15 \mathrm{~h}, 24 \mathrm{~h}, 31 \mathrm{~h}, 38 \mathrm{~h}, 48 \mathrm{~h}, 55 \mathrm{~h}, 62 \mathrm{~h}$, and $72 \mathrm{~h}$ after incubation. After incubation for $122 \mathrm{~h}, 7 \mathrm{w} / \mathrm{v} \%$ of aliquot 336 containing ethyl acetate was $(25 \mathrm{~mL})$ added to the production medium, and the $\mathrm{pH}$ of the mixture was adjusted to 10.5 with $5 \mathrm{M} \mathrm{NaOH}$. The broth was shaken vigorously at $200 \mathrm{rpm}$ for $1 \mathrm{~h}$, and the $\mathrm{pH}$ of the broth was adjusted to 10.5 with $5 \mathrm{M} \mathrm{NaOH}$. The organic layer was collected and stored at $4^{\circ} \mathrm{C}$ for 7 days. The crude skipped-13C-AmB was precipitated as yellow solids, and the crudes were collected by centrifugation, washed with acetone $(3 \mathrm{~mL} \times 2), \mathrm{MeOH}(2 \mathrm{~mL})$, 
and dried with Ar flow. The yellow solid was dissolved in DMF $(1 \mathrm{~mL})$ containing $0.2 \mathrm{~mL}$ of $\mathrm{AcOH}$ and precipitated by adding diethyl ether $(10 \mathrm{~mL})$. The precipitate was filtered with Celite and dissolved in $\mathrm{CHCl3} / \mathrm{MeOH} / \mathrm{H} 2 \mathrm{O}=10: 6: 1$ to afford purified skipped-13C-AmB $(8.9 \mathrm{mg})$. The purity was checked with $13 \mathrm{C}$ NMR. The average labeling rate of $13 \mathrm{C}$ was estimated to be $26 \%$ according to the isotope pattern of ESI mass spectra (Fig. M7, see Supplementary Materials).

\section{Membrane sample preparation}

Preparation of liposome sample for ${ }^{19} \mathrm{~F}$ CODEX: $14-\mathrm{F}-\mathrm{AmB}(1.98 \mathrm{mg}, 2.1 \mu \mathrm{mol})$, Erg $(2.50 \mathrm{mg}, 6.3$ $\mu \mathrm{mol})$, and POPC (11.2 mg, $14.7 \mu \mathrm{mol})$ were dissolved in $\mathrm{CHCl}_{3} / \mathrm{MeOH} .(1: 2, \mathrm{v} / \mathrm{v})$. The solvent was removed under reduced pressure and dried in vacuo for 8 hours. The lipid film was hydrated with $\mathrm{H}_{2} \mathrm{O}$ $(500 \mu \mathrm{l})$ and $10 \mathrm{mM}$ HEPES buffer $(14.0 \mu \mathrm{l})$ by vortexing, and the lipid dispersion was freeze-thawed 5 cycles. After transferring to an Eppendorf tube, water was removed by freeze-drying. After the powder was hydrated with $\mathrm{D}_{2} \mathrm{O}$ (14.0 $\mu 1,50 \%$ hydration), $300 \mu \mathrm{l}$ of $\mathrm{H}_{2} \mathrm{O}$ was added, followed by centrifugation at $15,000 \mathrm{rpm}$ for $10 \mathrm{~min}$, and then supernatant was removed by decantation. This operation was repeated three times. The obtained hydrated lipid membrane sample was transferred into a 4 mm MAS rotor (Bruker).

Preparation of liposome sample for ${ }^{13} \mathrm{C}\left\{{ }^{19} \mathrm{~F}\right\}$ REDOR of 14-F-AmB and skipped- ${ }^{13} \mathrm{C}-\mathrm{AmB}$ in POPCErg MLVs: $14-F-A m B / s k i p p e d-{ }^{13} \mathrm{C}-\mathrm{AmB}$, Erg and POPC (0.5: 0.5: 3: 7, total $\left.15 \mathrm{mg}\right)$ were dissolved in $\mathrm{CHCl}_{3} / \mathrm{MeOH}$. Then the solvent was removed by evaporation to make thin film, and the film was dried over in vacuo for $18 \mathrm{~h}$. After hydrated with $\mathrm{H}_{2} \mathrm{O}(1 \mathrm{~mL})$ and $10 \mathrm{mM}$ HEPES buffer $(\mathrm{pH} 7.0,15 \mu \mathrm{L})$, the lipid dispersion was freeze-thawed 5 times to prepare MLVs, and lyophilized. The powder thus obtained was rehydrated with $15 \mu \mathrm{L}$ of $\mathrm{D}_{2} \mathrm{O}$, and packed into a HR-MAS insert (Bruker, Germany). Then the insert was inserted into a $4 \mathrm{~mm}$ MAS rotor (Bruker).

Preparation of liposome sample for ${ }^{13} \mathrm{C}\left\{{ }^{19} \mathrm{~F}\right\}$ REDOR of 32-F-AmB, 26, $40-{ }^{13} \mathrm{C}_{2}$-AmB in POPC-Erg 
over in vacuo for $18 \mathrm{~h}$. After hydrated with $\mathrm{H}_{2} \mathrm{O}(0.5 \mathrm{~mL})$ and $10 \mathrm{mM}$ HEPES buffer $(\mathrm{pH} 7.0,20 \mu \mathrm{L})$, the lipid dispersion was freeze-thawed 5 times to prepare MLVs, and lyophilized. The powder thus obtained was rehydrated with $20 \mu \mathrm{L}$ of $\mathrm{D}_{2} \mathrm{O}$, and packed into a glass tube and sealed with epoxy glue. Then, the tube was inserted into a $5 \mathrm{~mm}$ MAS rotor (Varian).

\section{NMR spectral measurements}

Solid-state NMR measurements: Solid-state NMR spectra were recorded using a Bruker Avance III spectrometer equipped with a 4 mm HFX triple resonance probe or CMX300 (Chemagnetics) equipped with $5 \mathrm{~mm}$ HFC triple resonance probe (Varian). The ${ }^{19} \mathrm{~F}$ CODEX ${ }^{16,17}$ experiments were carried out with $3 \mathrm{kHz}$ of MAS at $0{ }^{\circ} \mathrm{C}$. The CSA evolution period was set to $0.67 \mathrm{~ms}$, and $66 \mathrm{kHz}$ of ${ }^{1} \mathrm{H}$ decoupling field strength was applied during the CSA evolution and FID acquisition period. The $90^{\circ}$ pulse width of ${ }^{19} \mathrm{~F}$ was $4.43 \mu$ s. The ${ }^{13} \mathrm{C}\left\{{ }^{19} \mathrm{~F}\right\}$ REDOR spectra were recorded with 5 or $7 \mathrm{kHz}$ MAS at 25 or $30^{\circ} \mathrm{C}$. The $90^{\circ}$ pulse width of ${ }^{1} \mathrm{H}$, the $180^{\circ}$ pulse width of ${ }^{19} \mathrm{~F}$, and $180^{\circ}$ pulse width of ${ }^{13} \mathrm{C}$ were $3.78 \mu \mathrm{s}, 10.8 \mu \mathrm{s}$, and $7.86 \mu$ s, respectively. $66 \mathrm{kHz}$ of ${ }^{1} \mathrm{H}$ decoupling field strength was applied during the dipole evolution and FID acquisition period. Static ${ }^{2} \mathrm{H}$ NMR spectra were recorded on a VNS300 (Varian) spectrometer equipped with a $5 \mathrm{~mm}$ static probe (Varian). ${ }^{2} \mathrm{H} 90^{\circ}$ pulse width was $2.5 \mu \mathrm{s}$, and pre and post echo delay times of solid-echo pulse sequence were 30 and $25 \mu$ s, respectively.

UV-VIS/CD spectral measurements: AmB and lipids were mixed into vials from the stock solution to a molar ratio of approximately 1:10, 1: 100, and 1: 1000 (10 mol\% or $30 \mathrm{~mol} \%$ of POPC was replaced with sterols in sterol-containing samples). The solvent was distilled off (V-10, Biotage, Sweden), and the lipid film obtained was dried in vacuum overnight. After hydration with $2 \mathrm{~mL}$ of $\mathrm{H}_{2} \mathrm{O}$ (MilliQ), freezethaw was repeated 3 times to obtain MLV. The MLV was passed through a polycarbonate filter (Liposofirst ${ }^{\circledR}$ ) with a pore size of $800 \mathrm{~nm}$ to yield LUVs. In order to determine the composition ratio after extrusion through the filter, $\mathrm{AmB}$ was quantified by dissolving $100 \mu \mathrm{L}$ of the LUV suspension in $900 \mathrm{uL}$ of DMSO and subjected to colorimetric determination by UV-VIS spectra. The POPC content was quantified by using the phospholipid C-test Wako kit (FUJIFILM Wako Pure Chemical Corporation, 
Japan), and the Cho and Erg contents were quantified using the cholesterol E-test Wako kit (FUJIFILM Wako Pure Chemical Corporation, Japan). From these quantitative values, the final ratio of AmB / sterol / POPC of each LUV sample was determined as described in the legends of Fig. S9 and S10. UV-VIS absorption spectra were recorded on a V-730BIO ((JASCO Corporation, Japan) at room temperature (22$25^{\circ} \mathrm{C}$ ) using a cell with an optical path length of $10 \mathrm{~mm}$. CD spectra were measured a J-720W (JASCO Corporation, Japan). The measurements were performed at $25^{\circ} \mathrm{C}$ using a cell with an optical path length of $2 \mathrm{~mm}$.

\section{Construction of AmB assembly based on REDOR dephasing curves}

The $x y z$ coordinate of $\mathrm{AmB}$ was constructed based on an X-ray structure ${ }^{22}$. Mycosamine orientation was modified according to our report ${ }^{23}$. The coordinates are summarized in Table S1. The AmB coordinates were then rotated by the Euler angle $(\alpha, \beta, \gamma)$ as follow:

$$
\left(\begin{array}{l}
x^{\prime} \\
y^{\prime} \\
z^{\prime}
\end{array}\right)=\left(\begin{array}{ccc}
\cos \gamma \cos \beta \cos \alpha-\sin \gamma \sin \alpha & -\sin \gamma \cos \beta \cos \alpha-\cos \gamma \sin \alpha & \sin \beta \cos \alpha \\
\cos \gamma \cos \beta \sin \alpha+\sin \gamma \cos \alpha & -\sin \gamma \cos \beta \sin \alpha+\cos \gamma \cos \gamma & \sin \beta \sin \alpha \\
-\cos \gamma \sin \beta & \sin \gamma \sin \beta & \cos \beta
\end{array}\right)\left(\begin{array}{l}
x \\
y \\
z
\end{array}\right)
$$

where, $(x, y, z)$ and $\left(x^{\prime}, y^{\prime}, z^{\prime}\right)$ are coordinates of AmB before and after Euler rotation, respectively. AmB coordinates were moved in parallel with the $x$-axis by $R$ (See Fig. S3 for the definition of $R$ as a pore radius):

$$
\left(\begin{array}{l}
x^{\prime \prime} \\
y^{\prime \prime} \\
z^{\prime \prime}
\end{array}\right)=\left(\begin{array}{l}
x^{\prime} \\
y^{\prime} \\
z^{\prime}
\end{array}\right)+\left(\begin{array}{l}
R \\
0 \\
0
\end{array}\right)
$$

and duplicated by rotating around the $z$-axis to form $C_{\mathrm{N}}$ symmetric channel assembly:

$$
\left(\begin{array}{l}
x_{k}^{\prime \prime \prime} \\
y_{k}^{\prime \prime \prime} \\
z_{k}^{\prime \prime \prime}
\end{array}\right)=\left(\begin{array}{ccc}
\cos (2 \pi k / N) & -\sin (2 \pi k / N) & 0 \\
\sin (2 \pi k / N) & \cos (2 \pi k / N) & 0 \\
0 & 0 & 1
\end{array}\right)\left(\begin{array}{l}
x^{\prime \prime} \\
y^{\prime \prime} \\
z^{\prime \prime}
\end{array}\right)
$$

where, $\left(x_{k}^{\prime \prime \prime}, y_{k}^{\prime \prime \prime}, z_{k}^{\prime \prime \prime}\right)$ is the coordinate of $k^{\text {th }}$ duplicate of AmB. The interatomic distance between $k^{\text {th }}$ and $(k+1)^{\text {th }}$, and $k^{\text {th }}$ and $(k-1)^{\text {th }} \mathrm{AmB}$ molecules were then calculated.

The REODR curves were calculated by considering both 14-F-AmB intramolecular natural abundance ${ }^{13} \mathrm{C}$ contribution and 14-F-AmB/skipped- ${ }^{13} \mathrm{C}-\mathrm{AmB}$ intermolecular contribution. The 
intramolecular REDOR dephasings were calculated based on the coordinates shown in Table M1. In the case of 14-F/C41 combination, the overall REDOR dephasing, $\left(\Delta S / S_{0}\right)_{C 41}$, was calculated as follows:

$$
\begin{aligned}
\left(\Delta S / S_{0}\right)_{C 41}= & \frac{X_{n}}{X_{n}+X_{e}}\left(\Delta S / S_{0}\right)_{\text {intra }} \\
& +\frac{X_{e}}{X_{n}+X_{e}}\left\{p(1-p)\left(\frac{\Delta S}{S_{0}}\right)_{r_{1}}+p(1-p)\left(\Delta S / S_{0}\right)_{r_{2}}+p^{2}\left(\Delta S / S_{0}\right)_{r_{12}}\right\}
\end{aligned}
$$

where, $X_{n}, X_{e}$, and $p$ are a ${ }^{13} \mathrm{C}$ ratio of natural abundance, a ${ }^{13} \mathrm{C}$-enriched ratio, and a probability parameter, respectively. The $\left(\Delta S / S_{0}\right)_{\text {intra }},\left(\Delta S / S_{0}\right)_{r 1}$, and $\left(\Delta S / S_{0}\right)_{r 2}$ are intramolecular REDOR dephasing and intermolecular REDOR dephasings depending on two neighboring distance $r_{l}$ and $r_{2}$, respectively (see Fig. 4A). They were calculated as 2-pin systems. The $\left(\Delta S / S_{0}\right)_{r l 2}$ is the REDOR dephasing depending on both $r_{1}$ and $r_{2}$, which was calculated as a 3 -spin system. Bessel function approximation ${ }^{31}$ was used for the REDOR dephasing curves of the 2-spin system, and 144 angle sets were used for the REDOR dephasing curves of the 3 -spin system.

In the case of the REDOR dephasing curve observed on $\mathrm{C} 1$ ' carbon, the natural abundance ${ }^{13} \mathrm{C}$ signal of the $\mathrm{C} 13$ position of skipped- ${ }^{13} \mathrm{C}-\mathrm{AmB}$ overlapped with that of $\mathrm{C} 1$ '. Therefore, both $\mathrm{C} 1$ ' and $\mathrm{C} 13$ contributions were taken into account. Similarly, in the case of the heptaene moiety, the signals from C20 to C33 positions largely overlapped. Therefore, each REDOR curves for overlapping carbons was calculated and summed up. To eliminate the REDOR dephasings caused by longer ${ }^{13} \mathrm{C}-{ }^{19} \mathrm{~F}$ spin pairs such as inter-channel interaction, the experimental data observed at less than $15 \mathrm{~ms}$ dephasing time (seven data points for each labelled position) were taken into account for channel structure screening. The best fit parameters were obtained by RMSD analysis.

$$
\mathrm{RMSD}=\sqrt{\frac{\sum_{i=1}^{7}\left\{\left(\Delta S / S_{0}\right)_{C 41, i}^{c a l c}-\left(\Delta S / S_{0}\right)_{C 41, i}^{\text {exp }}\right\}+\sum_{i=1}^{7}\left\{\left(\Delta S / S_{0}\right)_{C 1^{\prime}, i}^{\text {calc }}-\left(\Delta S / S_{0}\right)_{C 1^{\prime}, i}^{\text {exp }}\right\}+\sum_{i=1}^{7}\left\{\left(\Delta S / S_{0}\right)_{\text {hept }, i}^{\text {calc }}-\left(\Delta S / S_{0}\right)_{\text {hep }, i}^{\text {exp }}\right\}}{21}}
$$


where, $\left(\Delta S / S_{0}\right)_{C 41, i}^{c a l c},\left(\Delta S / S_{0}\right)_{C 1^{\prime}, i}^{c a l c}$ and $\left(\Delta S / S_{0}\right)_{C 1^{\prime}, i}^{c a l c}$ are the calculated REDOR dephasing ratios of $i$ th dephasing time, respectively. $\left(\Delta S / S_{0}\right)_{C 4, i}^{\text {exp }},\left(\Delta S / S_{0}\right)_{C 1^{\prime}, i}^{\text {exp }}$ and $\left(\Delta S / S_{0}\right)_{h e p, i}^{\text {exp }}$ are the observed REDOR dephasing ratios of $i$ th dephasing time, respectively.

Methods of molecular dynamics (MD) simulations (for figures and tables, see Supplementary Materials)

We have carried out a series of MD simulations of channels composed of different number of AmB monomers (Fig. M1A, see Supplementary Materials), where the initial configuration of the channel complexed with ergosterols, Erg (Fig. M1D), were generated using the results of solid-state $\mathrm{NMR}^{18,32}$ in a palmitoyl oleoyl phosphatidylcholine (POPC) (Fig. M1F) lipid membrane. We also performed MD simulations of channels made by $\mathrm{AmB}$ derivatives; i.e., AmB methyl ester (AME) and N-acetyl-AmB $\left(\mathrm{AmB}_{\mathrm{NHCOCH}}\right)$ in Fig. M1B/C. To examine the effect of orientation of AmB channels on the assembling behavior of AmB channels, we simulated the multiple AmB channels with parallel and antiparallel arrangements in POPC membrane. We also examined the ion channel activity of hexamer, heptamer, and octamer AmB channels in 1,2-diphytanoyl-sn-glycero-3-phosphocholine (DPhPC) lipid bilayer (Fig. M1G).

For the simulated systems, we examined the ion permeation through channels consisting of different numbers of AmB monomers. The details of the simulated systems are provided in Table M2. The parallel orientation of $\mathrm{AmB} / \mathrm{Erg}$ or $\mathrm{AmB} / \mathrm{Cho}$ channel structure to be consistent with previous experimental data ${ }^{32}$ is considered. On the basis of the solid-state NMR measurements (Fig. 3), AmB molecules were arranged

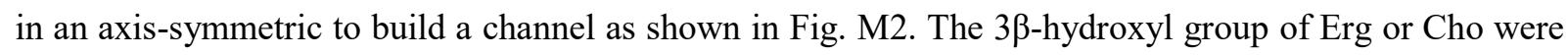
placed towards 2'-OH group of AmBs; which was reported as 'head-to-head' binding conformation ${ }^{32,33}$. The generated channel was embedded in a lipid bilayer for the system-V, sytem-VI-1, the system-VII-1 4, and the system-VIII-1 (Table M2), as shown in Fig. M3. We inserted a single AmB channel in the 'interdigitated' lipid membrane composed of $16 \mathrm{POPC}$ molecules $\left(\mathrm{AmB}_{5}, \mathrm{AmB}_{6}\right.$ and $\left.\mathrm{AmB}_{7}\right)$ or $32 \mathrm{POPC}$ 
(1-palmitoyl-2-oleoyl-sn-glycero-3-phosphocholine) $\left(\mathrm{AmB}_{8}\right)$ molecules in triclinic unit cell. The interdigitated lipid membrane was generated by arranging interdigitated POPC molecules.

We embedded nine modelled AmB-Erg channel complexes in the lipid membrane composed of 450 POPC molecules. This resulted in the same molecular ratio as in the experimental setup (AmB/Erg/POPC, 1: 3: 7) (System-VII-5; Fig. S15A). The configuration of the additional lipid membrane was generated using the CHARMM-GUI ${ }^{34-37}$. In the initial structures of system-VI-2, system-VII-7 and system-VIII-2 (Table M2), single $A_{m B}, A m B_{7}$, and $A_{m B}$ channels embedded in DPhPC lipid membrane, respectively, and surrounded by $\sim 14500$ water molecules and ions $\left(\sim 550 \mathrm{~K}^{+}, \sim 550 \mathrm{Cl}^{-}\right)$, resulting in the salt concentration of 2 M, using the CHARMM-GUI webserver. The initial structures are shown in Fig. M4. The similar salt concentration was chosen as in a single $\mathrm{AmB}$ channel conductance measurement in $\mathrm{DPhPC}^{24}$. The ten different initial structures of the System-VI-2, 16 different initial structures of the System-VII-7 and ten different initial structures of the System-VIII-2 were constructed by using CHARMM-GUI ${ }^{34-37}$.

In the case of AmB derivatives, we prepared the systems by replacing all AmB molecules of the SystemVII-1 to each AmB derivative shown in Figs. M1(B) and M1(C), respectively (the System-VII-3 4). We also set up the system by placing parallel and anti-parallel arrangement of AmB channels (System-VII-6) (Fig. M5).

To check the dependency on the initial conditions, we constructed 'ten' different lipid membrane structures including the AmB-sterol complex for the System-V, System-VI-1, System-VII-1 4, and System-VIII-1. For the System-VII-3 and System-VII-4 (Table M2), seven counter-ions $\left(\mathrm{Cl}^{-}\right)$and seven counter-ions $\mathrm{K}^{+}$were added to neutralize $\mathrm{AmB}$ derivatives, respectively. In the case of the System- $\mathrm{V}$, System-VI-1, System-VII-1 4, and System-VIII-1 (Table M2), $7 \mathrm{~K}^{+}$and $7 \mathrm{Cl}^{-}$ions were added to the equilibrated systems after 100-ns MD runs, to realize physiological salt concentration. The GROMACS "genion" tool was used to replace bulk water molecules with $\mathrm{K}^{+}$and $\mathrm{Cl}^{-}$ions. The final number of molecules in each system is given in Table M2. 
30. Matumori, N. et al. Direct interaction between amphotericin B and ergosterol in lipid bilayers as revealed by ${ }^{2}$ H NMR spectroscopy. J. Am. Chem. Soc. 131, 11855-11860 (2009).

31. Mueller.K. T. Analytic solution for the time evolution of dipolar-dephasing NMR signals. J. Magn. Reason. Ser. A 113, 81-93 (1995).

32. Umegawa,Y., Matsumori, N., Oishi, T. \& Murata. M. Ergosterol increases the intermolecular distance of amphotericin B in the membrane-bound assembly as evidenced by solid-state NMR. Biochemistry 47, 1346313469 (2008).

33. Umegawa, Y. et al. Head-to-tail interaction between amphotericin B and ergosterol occurs in hydrated phospholipid membrane. Biochemistry 51, 83-89 (2012).

34. Jo, S., Lim, J. B., Klauda, J. B. \& Im, W. CHARMM-GUI membrane builder for mixed bilayers and its application to yeast membranes. Biophys. J. 97, 50-58 (2009).

35. Wu, E. L. et al. CHARMM-GUI membrane builder toward realistic biological membrane simulations. $J$. Comput. Chem. 35 1997-2004 (2014).

36. Lee, J. et al. CHARMM-GUI input generator for NAMD, GROMACS, AMBER/OpenMM, and CHARMM/OpenMM simulations using the CHARMM36 additive force field. J. Chem. Theory Comput. 12, 405-413 (2016).

37. Jo, S., Kim, T., Iyer, V. G. \& Im, W. CHARMM-GUI: A web-based graphical user interface for CHARMM. J. Comput. Chem. 29, 1859-1865 (2008).

Acknowledgments: The calculations were performed using the facilities of the supercomputer center at the Research Center for Computational Science (Okazaki, Japan) and the Institute for Solid State Physics, The University of Tokyo (Tokyo, Japan).

Funding:

JSPS KAKENHI Grant Numbers 16H06315 (UY, MD, WS, MM)

JSPS KAKENHI Grant Numbers 17H06406 (HT, MM)

JST ERATO Lipid active structure project JPMJER1005, (UY, MM) 


\section{Author contributions:}

Project conception by M.M., N. M. and W.S

Syntheses of labelled-AmBs by Y. U., T. Y., Y. N., H. T., T. S. and S. M.

Synthetic strategy by H. T., S. H., and T. O.

NMR measurements by Y. U., T. Y., Y. N., T. S. and S. M.

NMR data interpretation by Y. U., T. Y., T. S. and M. M.

MD simulations by M. D., K. F., S. S. and W. S.

Comprehensive analysis of ion channel structure and function by Y. U., T. Y., M. D., Y. N., W. S. and M. M.

Competing interests: The authors declare no competing interests.

\section{Supplementary Information}

Correspondence and requests for materials should addressed to Michio Murata or Wataru Shinoda. 